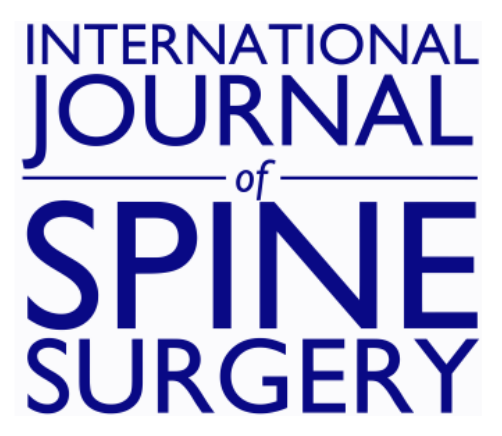

\title{
Full Endoscopic Lumbar Discectomy Versus Laminectomy for Cauda Equina Syndrome
}

Liu Yankang, Zhang Leiming, Kai-Uwe Lewandrowski, Tang Xiangyu, Zhu Zexing, Xu Jianbiao, Zhang Lin, Yuan Heng and Zhang Xifeng

Int J Spine Surg 2021, 15 (1) 105-112

doi: https://doi.org/10.14444/8014

http://ijssurgery.com/content/15/1/105

This information is current as of April 26, 2023.

Email Alerts Receive free email-alerts when new articles cite this article. Sign up at:

http://ijssurgery.com/alerts

The International Journal of Spine Surgery

2397 Waterbury Circle, Suite 1,

Aurora, IL 60504, Phone: +1-630-375-1432 


\title{
Full Endoscopic Lumbar Discectomy Versus Laminectomy for Cauda Equina Syndrome
}

\author{
LIU YANKANG, MD, ${ }^{1}$ ZHANG LEIMING, $\mathrm{MD},{ }^{2} \mathrm{KAI-UWE} \mathrm{LEWANDROWSKI,} \mathrm{MD,}{ }^{3}$ \\ TANG XIANGYU, MD, ${ }^{4}$ ZHU ZEXING, MD ${ }^{4}$ XU JIANBIAO, MD,${ }^{5}$ ZHANG LIN, MD,${ }^{6}$ \\ YUAN HENG, MD, ${ }^{1}$ ZHANG XIFENG, $\mathrm{MD}^{4}$ \\ ${ }^{I}$ Shanxi Medical University, Taiyuan, China, ${ }^{2}$ Department of Neurosurgery, The Sixth Medical Center of PLA General Hospital, Beijing, China, ${ }^{3}$ Center for \\ Advanced Spine Care of Southern Arizona and Surgical Institute of Tucson, Tucson, Arizona, Department of Orthopaedics, Fundación Universitaria Sanitas, \\ Bogotá, D.C., Colombia, ${ }^{4}$ Spine Division of Orthopaedic Department, PLA General Hospital, Beijing, China, ${ }^{5}$ School of Clinical Medicine, Tsinghua University, \\ Beijing, China, ${ }^{6}$ Department of Orthopedics, Branch of PLA General Hospital, Sanya, China
}

\begin{abstract}
Background: Typically, open surgery is advocated for cauda equina patients. The goal of this study was to compare the clinical efficacy of full endoscopic lumbar discectomy and laminectomy in the treatment of cauda equina syndrome (CES) caused by lumbar disc herniation.

Methods: Forty-three patients with CES either underwent endoscopic or laminectomy surgery from May 2015 to April 2016, and data were collected and retrospectively analyzed. The patients were divided into 2 groups according to the surgical methods: the endoscopy group (with 21 patients, 14 males and 7 females, and an average age of 42.67 with a standard deviation of 9.70 years) and the laminectomy group (with 22 patients, 16 males and 6 females, and an average age of 44.55 with a standard deviation of 9.36 years). The modified Japanese Orthopaedic Association (JOA) "legtrunk-bladder" score was used to assess the efficacy of the respective surgical methods.

Results: Analysis showed longer surgery time, more bleeding, and longer hospital stay in the laminectomy group than in the endoscopy group with statistical significance. The postoperative JOA scores improved in both groups when compared with those before the operation, and the differences were statistically significant. There were no significant differences in JOA scores between the 2 groups at preoperation and 6-month and 1-year follow-ups. There was 1 patient in each group whose CES symptoms worsened after endoscopy. However, immediate reoperation resulted in satisfactory outcomes.

Conclusions: CES clinical symptom resolution was equal with endoscopy and laminectomy both in short-term and midterm follow-up. However, endoscopic treatment was advantageous by reducing the amount of bleeding, duration of surgery, and hospitalization days when compared to laminectomy.
\end{abstract}

Level of Evidence: 3.

Clinical Relevance: Feasibility study Endoscopic Decompression for Cauda Equina

Minimally Invasive Surgery

Keywords: cauda equina syndrome, lumbar disc herniation, laminectomy, full endoscopic decompression

\section{INTRODUCTION}

Cauda equine syndrome (CES) is the result of acute nerve root compression below the conus of the spinal cord. Motor and sensory function to the lower extremities and bladder may be disrupted. Often multiple lumbar and sacral nerve roots are involved. The symptoms are excruciating and often prompt admission of the patient to the hospital for emergent decompression. CES can lead to incontinence and even permanent paralysis.

According to Fraser et $\mathrm{al}^{1}$ the diagnosis of CES is made if the patient has one or more of the following symptoms: (1) bladder or rectal dysfunction, (2) sensory dysfunction in the saddle area, (3) sexual dysfunction, or (4) lower extremity pain and motor weakness. The most common cause of CES is lumbar disc herniation (LDH). ${ }^{1-3}$ CES can be divided into incomplete CES and complete CES. While the latter is defined by the complete loss of bladder and rectal function, incomplete CES patients were divided by Shi et $\mathrm{al}^{4}$ into groups based on clinical features as follows: (1) preclinical, (2) early, (3) middle, and (4) late. These different stages are characterized by various degrees of decreased motor strength and sensory dysfunction. Early surgical decompression is still considered the gold standard by most authors. ${ }^{1,2,5-7}$ Traditionally, total 
laminectomy was the procedure of choice for surgical decompression in CES patients. ${ }^{7-9}$ More recent studies have reported satisfactory results of hemilaminectomy for the disease. ${ }^{2,10}$ Other authors have considered endoscopic surgery as an alternative method, but there are only a few case reports of full endoscopic treatment of LDH in CES patients in the existing literature. ${ }^{11-13}$

In this study, the authors investigated the feasibility of full endoscopic lumbar discectomy, particularly in patients with incomplete CES, and compared its efficacy to traditional open laminectomy. Moreover, the authors aimed to describe the indications and complications of full endoscopic CES decompression for LDH as the current clinical evidence in this area is scarce.

\section{MATERIALS AND METHODS}

\section{Patients and CES Staging}

From May 2015 to April 2017, 43 patients with early and mid-stage CES secondary to LDH were surgically treated treatment in the first author's hospital. Patients were treated either with endoscopic surgery (endoscopy group), or laminectomy (open surgery group). The application of the inclusion and exclusion criteria described below resulted in the enrollment of 21 patients in the endoscopy group, which consisted of 14 males and 7 females with an average age of 42.67 with a standard deviation (SD) of 9.70 years. The laminectomy group included 22 patients comprised of 16 males and six females with an average age of 44.55 and SD of 9.36 years. The endoscopy patients underwent surgery under local anesthesia with sedation using monitored anesthesia care protocols, and the laminectomy patients underwent surgery under general anesthesia.

Patients' clinical presentation was assessed based on their features and timing of onset of symptoms according to Shi et $\mathrm{al}^{4}{ }^{4}$ who suggested grouping patients into the following 4 groups:

Group 1 (preclinical): low back pain with only bulbocavernosus reflex and ischiocavernosus reflex abnormalities. Group 2 (early): saddle sensory disturbance and bilateral sciatica. Group 3 (middle): saddle sensory disturbance, bowel or bladder dysfunction, motor weakness of the lower extremity, and reduced sexual function. Group 4 (late): the absence of saddle sensation and sexual function in addition to uncontrolled bowel function. ${ }^{4}$
This group assignment was used in the crosstabulation statistical analysis described below.

\section{Inclusion and Exclusion Criteria}

The inclusion criteria were as follows:

(1) diagnosis of CES caused by LDH, with clinical symptoms limited to early- and midstage;

(2) complete clinical and imaging follow-up data; and

(3) minimum follow-up time of 1 year.

There were 7 exclusion criteria:

(1) presence of peripheral neuropathy or any other nerve disease,

(2) spinal fracture,

(3) central spinal canal stenosis $<100 \mathrm{~mm}^{2}$ crosssectional area at the surgical level,

(4) infection,

(5) tumor,

(6) recent spinal surgery within the last year, and

(7) late cauda equina syndrome with severe pain, motor weakness, and overt urinary retention.

\section{Surgical Techniques}

Laminectomy with discectomy was done through a midline incision approximately $7 \mathrm{~cm}$ in length in standard fashion with subperiosteal dissection, removal of the lamina with the detachment of the supraspinous ligament with the removal of the spinous process and lamina. A wide laminectomy was frequently accompanied with some resection of the medial portion of the facet joints while being conscientious of avoiding excessive lateral resection of the facet joint to minimize the risk of postlaminectomy instability.

In comparison, during the endoscopic decompression, the authors limited the irrigation pump pressure to less than $40 \mathrm{~mm} \mathrm{Hg}$ and performed a foraminoplasty to increase the neuroforaminal volume by abrading parts of the superior articular process in a posterior direction and part of the vertebral ring apophysis of the lower vertebral body. In 7 cases of associated lateral recess stenosis due to facet or ligamentum flavum hypertrophy or very large upward-migrated disc herniations (Figure 1), the interlaminar approach was employed to perform a unilateral or bilateral partial abrasion of the lamina and medial aspect of the facet joint. During 

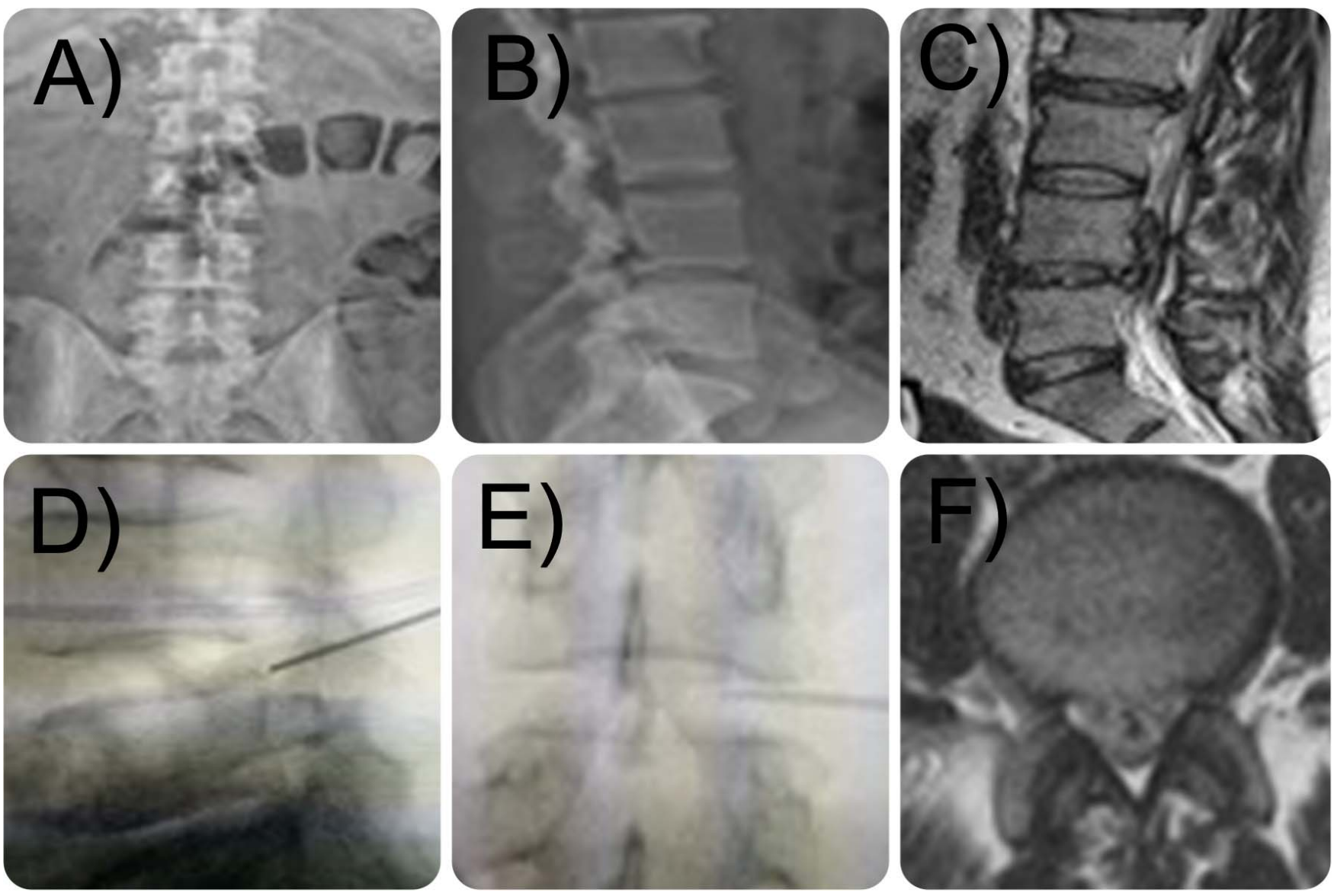

Figure 1. An exemplary case of a 35-year-old man with saddle anesthesia, fecal incontinence, decreased sensation and pain in the buttocks and lower right knee, and right foot drop for 7 days. The patient's symptoms improved significantly within days following percutaneous endoscopic lumbar discectomy (PELD) decompression. The preoperative (A) posteroanterior and (B) lateral radiographs. The preoperative (D) sagittal and (F) axial magnetic resonance imaging scans show a large upward-migrated extruded disc herniation. The intraoperative fluoroscopic (D) lateral and (E) posteroanterior views of the access needle positioning during the transforaminal PELD are shown.

the interlaminar approach, the working channel was inserted into the lateral recess after the removal of the ligamentum flavum. The discectomy was performed after the intervertebral disc was sufficiently exposed. Postoperative surveillance imaging studies were typically taken at 6 months postoperatively to monitor resolution of dural sac compression (Figure 2).

\section{Patient Follow-Up and Outcome Measures}

Perioperative data included intraoperative blood loss, length of the operation, length of hospital stay, operation-related complications, and reoperation. The authors also recorded if there were any complications, including nerve root injury, dural tear, or incomplete decompression. The modified Japanese Orthopaedic Association (JOA) "legtrunk-bladder" score was used to evaluate the recovery of the neurological function by comparing preoperative numbers to those scored at 6 and 12 months postoperatively. A JOA improvement rate was calculated as a percentage as follows:

JOA improvement rate

$$
\begin{aligned}
= & \frac{\text { postoperative JOA score }- \text { preoperative JOA score }}{11-\text { preoperative JOA score }} \\
& \times 100 \text {. }
\end{aligned}
$$

This JOA improvement percentage rate was employed by the authors to define the patients' overall clinical outcomes by defining them as excellent (JOA rate of $76 \%-100 \%$ ), good (JOA rate of $51 \%-75 \%$ ), fair (JOA rate of $26 \%-50 \%$ ), and poor (JOA rate of $<26 \%$ ).

\section{Statistical Analysis}

IBM SPSS Statistics version 22.0 statistical software (IBM, Chicago, IL) was used for our data analysis. Data distribution was tested for normality, and means and standard deviations of all variables were calculated and tested for statistical significance 

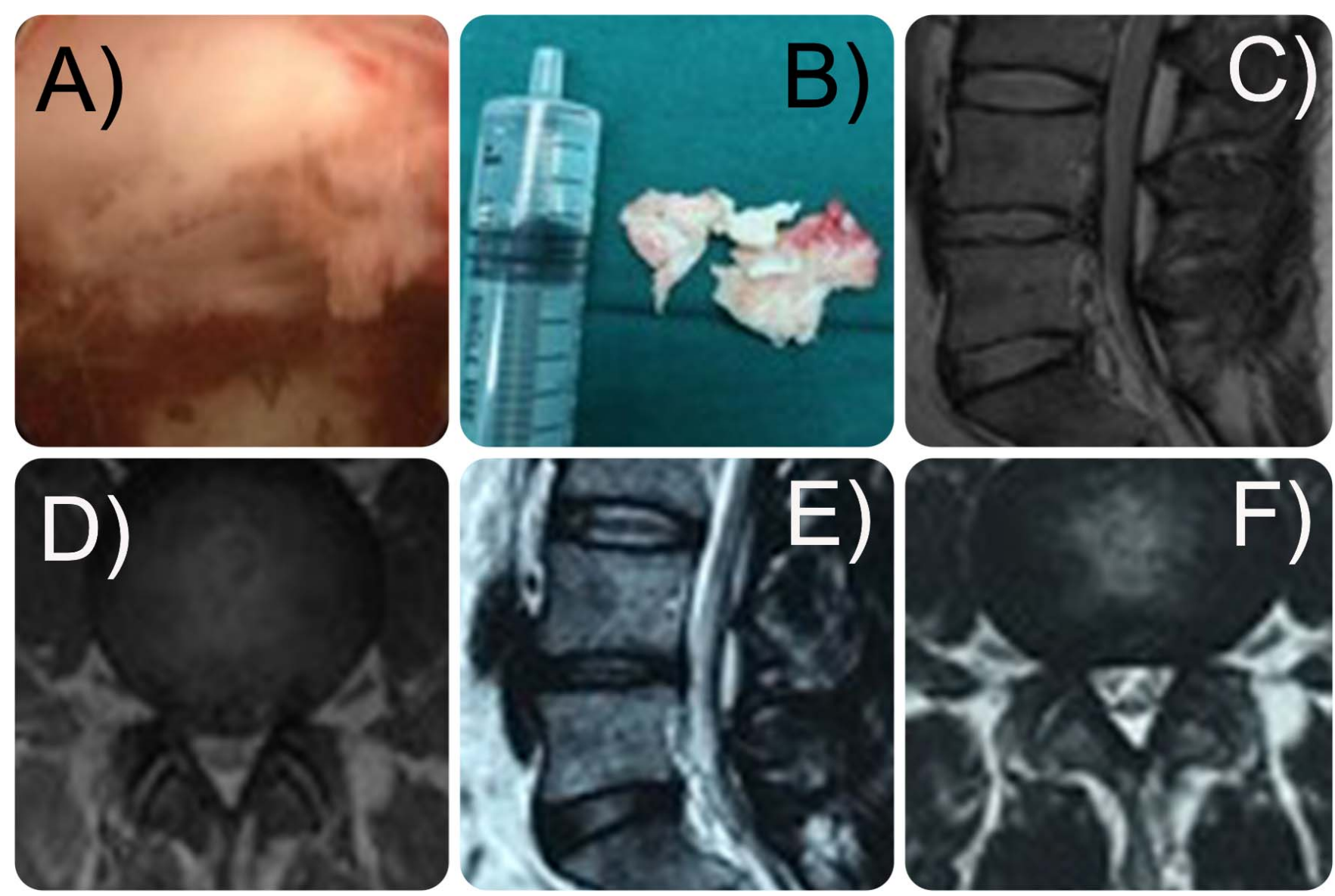

Figure 2. (A) Intraoperative direct view of the decompressed lumbar disc herniation of the exemplary case of a 35-year-old man with cauda equina syndrome shown in Figure 1. (B) Several large fragments of extruded disc herniations were removed endoscopically with percutaneous endoscopic lumbar discectomy (PELD). The postoperative (C) sagittal and (D) axial magnetic resonance imaging scans taken (E) 3 days and (F) 6 months after PELD surgery confirm adequate decompression and absence of recurrence.

employing a $P$ value of .05 using the Student $t$ test for independent samples. The difference analysis between ranks was done with the rank-sum test, expressing data count as a composition ratio. Chisquare testing was used for cross-tabulation comparisons of the clinical outcome categories versus the type of surgery.

\section{RESULTS}

Between the 2 surgical groups, there was no statistically significant difference in patients' age, gender, clinical stage, symptoms and signs, pathological segment, medical history, delay to surgery over 48 hours, or follow-up time (Table 1). However, there were statistically significant differences between the 2 groups with lower estimated intraoperative blood loss, length of operation, and hospitalization days (all $P<.001$ ).

Repeated analysis of variance analysis showed that the JOA score improvements obtained on patients undergoing either the endoscopic lumbar discectomy or laminectomy treatment were statistically significant $(F=257.320, P<.001)$ when comparing preoperative to postoperative numbers. However, there was no statistically significant difference between the groups $(F=0.531, P=$ $.470)$, or timing of surgery in either group $(F=$ $0.163, P=.702$; Table 2). Furthermore, there was no statistically significant difference in JOA scores between the 2 groups preoperatively, or postoperatively at 6-month and 1-year follow-up (both $P<$ .5). Clinical outcome analysis by the normalized JOA improvement scores in endoscopy patients showed excellent in 11 cases, good in 7 cases, and fair results in 3 cases. None of the 43 study patients had a poor outcome. Consequently, excellent and good outcome ratings were obtained $85.7 \%$ in the endoscopy group. In the laminectomy group, there were 9 cases with excellent, and another 9 cases with good outcomes ratings, rendering the success rate in the laminectomy group at $81.8 \%$ (Table 3 ). The 
Table 1. Demographic and clinical data of patients treated with either laminectomy or in percutaneous endoscopic lumbar discectomy.

\begin{tabular}{|c|c|c|c|c|c|}
\hline Parameter & & $\begin{array}{l}\text { Laminectomy } \\
\quad(\mathrm{n}=\mathbf{2 2})\end{array}$ & $\begin{array}{l}\text { Full Endoscopy } \\
\quad(\mathrm{n}=\mathbf{2 1})\end{array}$ & $t / z / x^{2}$ & $P$ \\
\hline Age $( \pm \mathrm{SD}$, range, in years $)$ & & $44.55 \pm 9.36$ & $42.67 \pm 9.70$ & 0.647 & .522 \\
\hline$N(\%)$ Female & & $6(27.3 \%)$ & $7(33.3 \%)$ & 0.187 & .665 \\
\hline \multirow[t]{3}{*}{ CES clinical period } & & & & 0.559 & .455 \\
\hline & Early & $8(36.4 \%)$ & $10(47.6 \%)$ & & \\
\hline & Intermediate & $14(63.6 \%)$ & $11(52.4 \%)$ & & \\
\hline \multirow[t]{4}{*}{ Spinal CES level } & & & & 1.879 & .537 \\
\hline & $\mathrm{L} 3 / 4$ & $0(0 \%)$ & $2(9.5 \%)$ & & \\
\hline & $\mathrm{L} 4 / 5$ & $11(50.0 \%)$ & $10(47.6 \%)$ & & \\
\hline & L5S1 & $11(50.0 \%)$ & $9(42.9 \%)$ & & \\
\hline \multirow[t]{5}{*}{ Main clinical symptom } & Low back pain & $17(77.3 \%)$ & $17(81.0 \%)$ & 0.000 & 1.000 \\
\hline & Saddle anesthesia & $12(54.5 \%)$ & $12(57.1 \%)$ & 0.029 & .864 \\
\hline & Decrease saddle sensation & $15(68.2 \%)$ & $11(52.4 \%)$ & 1.122 & .289 \\
\hline & Bladder and bowel dysfunction & $14(63.6 \%)$ & $14(66.7 \%)$ & 0.043 & .835 \\
\hline & Sexual dysfunction & $3(13.6 \%)$ & $0(0 \%)$ & 1.336 & .248 \\
\hline \multirow[t]{2}{*}{ Clinical course } & Medical decline & $10(45.5 \%)$ & $8(38.1 \%)$ & 0.239 & .625 \\
\hline & Weak reflexes & $19(86.4 \%)$ & $17(81.0 \%)$ & 0.005 & .946 \\
\hline Duration of symptoms in days ( $\pm \mathrm{SD}$, range) & & $47.91 \pm 14.24$ & $49.62 \pm 19.91$ & 0.325 & .747 \\
\hline Delayed surgery $(>48 \mathrm{~h})$ & & $20(90.9 \%)$ & $18(85.7 \%)$ & 0.003 & .956 \\
\hline Follow-up in months ( $\pm \mathrm{SD}$, range $)$ & & $15.47 \pm 2.25$ & $15.16 \pm 1.98$ & 0.479 & .635 \\
\hline Estimated blood loss (ml) & & $150(98,200)$ & $5(5,5)$ & 5.764 & $<.001$ \\
\hline Length of surgery in hours ( $\pm \mathrm{SD}$, range) & & $3.09 \pm 0.39$ & $0.69 \pm 0.15$ & 26.925 & $<.001$ \\
\hline Hospital stay in days $( \pm \mathrm{SD}$, range) & & $8.82 \pm 2.34$ & $5.00 \pm 1.95$ & 5.794 & $<.001$ \\
\hline \multirow[t]{5}{*}{ Surgical complications } & & $1(4.5 \%)$ & $1(4.8 \%)$ & 0.000 & 1.000 \\
\hline & Nerve root injury & $0(0 \%)$ & $0(0 \%)$ & & \\
\hline & Dural tear & $0(0 \%)$ & $0(0 \%)$ & & \\
\hline & Tethering and adhesions & $1(4.5 \%)$ & $0(0 \%)$ & & \\
\hline & Incomplete decompression & $0\left(0 \%{ }^{\wedge}\right)$ & $1(4.8 \%)$ & & \\
\hline Reoperation & & $1(4.5 \%)$ & $1(4.8 \%)$ & 0.000 & 1.000 \\
\hline
\end{tabular}

Abbreviation: CES, cauda equina syndrome.

remaining $18.2 \%$ in the laminectomy group had fair ( 3 cases), and poor ( 1 case) outcomes.

Surgery-related complications occurred in both groups. One patient in the endoscopic group had difficulty in urinating on the same day after the operation. Reexamination of magnetic resonance imaging revealed residual intervertebral disc tissue in the spinal canal, and rapid endoscopic reoperation was performed. The residual tissue was removed during the operation, decompression was completed, and the postoperative recovery was good. One patient in the open laminectomy group had progressive worsening of urination 1 month after surgery, and emergency surgical exploration revealed nerve tethering with adhesions. Revision decompression and neurolysis did resolve the symptoms postoperatively.

Table 2. Comparison of JOA scores endoscopy and laminectomy patients by follow-up.

\begin{tabular}{lccc}
\hline $\begin{array}{l}\text { Treatment } \\
\text { and Statistics }\end{array}$ & Preoperative & 6 Months Postop & 1 Year Postop \\
\hline Laminectomy & $6.43 \pm 1.16$ & $9.50 \pm 0.86 \mathrm{a}$ & $9.64 \pm 0.83 \mathrm{a}$ \\
Endoscopy & $6.50 \pm 1.13$ & $9.74 \pm 1.06 \mathrm{a}$ & $9.86 \pm 0.95 \mathrm{a}$ \\
$t$ & 0.195 & 0.813 & 0.811 \\
$P$ & .846 & .421 & .422 \\
\hline
\end{tabular}

\section{DISCUSSION}

The prognosis of CES is dependent on multiple factors such as the duration of time leading up to decompression surgery, medical history, the severity of symptoms at the time of presentation, and the size and location of the compression pathology. There is consensus in the literature in support of better prognosis with early intervention in incomplete CES. ${ }^{5,8,11}$ Qureshi and Sell et al ${ }^{14}$ reported that the severity of preoperative urinary dysfunction is a crucial prognosticator of recovery of function after an intervention. All patients included in this study presented with early-onset CES or with symptoms of midterm duration. Therefore, authors had better control of the management of contributing risk factors in patients in the 2 study groups - full endoscopic decompression and laminectomy decompression - that could ultimately affect clinical

Table 3. Clinical outcomes for endoscopy and laminectomy.

\begin{tabular}{lrrrr}
\hline $\begin{array}{l}\text { Treatment } \\
\text { and Statistics }\end{array}$ & Excellent & Good & Poor & Difference \\
\hline Laminectomy & $9(40.9 \%)$ & $9(40.9 \%)$ & $3(13.6 \%)$ & $1(4.5 \%)$ \\
Endoscopy & $11(52.4 \%)$ & $7(33.3 \%)$ & $3(14.3 \%)$ & $0(0 \%)$ \\
$Z$ & & 0.753 & \\
$P$ & & .451 & \\
\hline
\end{tabular}


outcomes. Since there were no patients with late CES in this study, the authors recommend continuing to treat the highly irritated neural elements in those patients with wide-open decompression until a formal analysis of this particular subgroup of patients can be done. Results of the statistical analysis did not show any significant differences in clinical outcome measures between the endoscopy and laminectomy group, and overall the clinical outcomes seem largely very comparable. JOA scores were significantly improved at the 6-month and 1year postoperative follow-ups for both laminectomy and endoscopy, suggesting that either of these 2 decompression surgeries are reasonable choices for CES patients with an underlying LDH pathology. The authors found equivalent timeliness to surgery data with little delay, and high efficacy in improving nerve function.

At 1 year follow-up, excellent and good results according to the JOA improvement rate were achieved in $85.7 \%$ of patients who underwent endoscopy compared to $81.8 \%$ in the laminectomy group. Prior reports in the laminectomy literature corroborate these favorable outcome numbers. Gleave and Macfarlane et $\mathrm{al}^{8}$ reported on 33 cases of acute urinary retention in CES patients who underwent laminectomy. At the final follow-up, $79 \%$ of their patients claimed that the bladder function was completely restored, but only $22 \%$ of the patients had complete resolution of sensory defects in the limbs or saddle area. Shapiro et al ${ }^{11}$ performed a laminectomy on 14 CES patients. Results of the last follow-up showed that $28 \%$ of patients still suffered from persistent urinary incontinence and fatigue. Dinning and Schaeffer ${ }^{9}$ treated 22 CES patients with bladder and/or rectal dysfunction with laminectomy, of which 17 patients $(77.3 \%)$ eventually returned to normal rectal function. The results of the authors' study corroborate these reported findings with $81.2 \%$ of our endoscopy patients having been graded as excellent. Hence, the authors conclude that in patients with early-onset and midstage CES full endoscopic decompression can be considered as an effective alternative to open laminectomy.

In this study, the differences in the length of operation, hospital stay, and estimated blood loss between endoscopy and laminectomy groups were statistically significant. An adequate intervertebral disc resection under endoscopy has the potential to reduce the risk for segmental instability caused by the operation because of reduced tissue trauma and preservation of the supraspinous ligamentous and facet joint complex. Moreover, the endoscopic surgery affords better direct visualization of the LDH. The combination of different endoscopic approaches allows a $360^{\circ}$ decompression with a more accurate definition of the surgical field than under microscopic visualization during laminectomy. The endoscopic working channel can also double-function as a retractor and safely retract the dural sac and nerve roots during the endoscopic decompression surgery. During a laminectomy, the damage to the lumbar motion segment due to the necessary removal of the posterior elements and partial facet joint resection may be extensive before the intervertebral disc is even exposed. The ensuing postoperative instability is collateral damage to the surgical exposure and is not dictated by the underlying disease. Also, endoscopic decompression can be done under local anesthesia and sedation, whereas laminectomy typically requires the patient to be under general anesthesia. More time to postoperative narcotic independence and more extended hospital stays are also inherent to laminectomy when compared to endoscopy. The analysis of short- and long-term complications revealed the absence of postoperative spondylolisthesis within the available follow-up period. Although rare, there were some complications. Nerve root tethering due to postoperative adhesions occurred in 1 case $(4.5 \%)$ in the laminectomy group and was discovered during open re-exploration. Another case $(4.8 \%)$ in the endoscopy group had incomplete decompression. The latter patient also underwent endoscopic revision decompression.

Managing CES patients postoperatively can be tricky, and it is not always easy to identify a reason for the increased symptoms after surgery. Clinical judgment is of utmost importance to avoid overtreatment on the back end of the treatment cycle. However, possible reasons for increased symptoms after CES decompression may be incomplete removal of the offending pathology, tearing of the dura mater, nerve root edema, nerve adhesions and tethering, a reperfusion injury, and last but not least, an overreaction of the highly irritated and inflamed lumbar and sacral nerve roots. Improper selection of surgical indications and weak grasp of clinical signs, inaccurate preoperative evaluation, and surgeons who lack the skill level to operate may increase the risk of surgery and lead to complica- 
tions. Endoscopic surgery uses local anesthesia and sedation, and the patient is under monitored anesthesia care, requiring the surgeon to pay attention to feedback from the patient obtained during surgery, helping the surgeon to avoid any inadvertent physical damage to the nerve structure. ${ }^{15}$ In the authors' opinion, endoscopic decompression if applied correctly in skilled hands may deliver favorable clinical results in the vast majority of CES patients while performing safely and efficiently with low risks of intraoperative and iatrogenic nerve root injury, particularly with the inside-out technique where the working cannula is inside the disc for most of the surgery. Thus, nerve root manipulation is minimal since the surgeon does not work in the epidural space. The entire process is far more complicated with laminectomy due to the need for more complex surgical setup, general anesthesia, and, in select cases, nerve monitoring to prevent iatrogenic nerve injury because nerve roots may be substantially retracted giving rise to the possibility of postoperative neuropraxia or even paralysis in worst cases. Dinning and Schaeffer ${ }^{9}$ advocated for open laminectomy to minimize compression of the already highly irritated dural sac and its contents. In comparison, there is only a report of one case by $\mathrm{Li}$ et $\mathrm{al}^{16}$ of decreased motor strength following endoscopy due to recurrent herniation.

A study of Jensen et $\mathrm{al}^{17}$ pointed out through a literature review that postoperative imaging of CES patients often shows substantial residuals of large disc herniations suggesting that a threshold for emergent surgical re-exploration should be low if symptoms worsen postoperatively without an apparent reason. Although the authors' study lacks statistical power to conclusively prove it, their experience is that there is an optimum time window for endoscopic surgical decompression within 48 hours of the patient's presentation to the emergency department. If missed, poor prognosis is more likely. This is in contrast to consensus reports on open laminectomy CES studies that concluded that there is no difference in prognosis between emergency surgery and delayed surgery. ${ }^{14,18,19}$ Therefore, further investigation of the importance of timeliness of surgical decompression with endoscopic versus open surgery should be further examined. The authors recommend a postoperative magnetic resonance imaging scan in the immediate postoperative period after endoscopic CES treatment to assess the size of the residual disc herniation, especially for those patients who have no signs of improvement.

\section{CONCLUSIONS}

Clinical improvements in CES patients were similar regardless of whether the decompression was done with open laminectomy or full endoscopic decompression. Endoscopic decompression of LDH in patients who develop symptoms early in the cauda equina syndrome can be considered as an alternative to open laminectomy. The reduced access trauma with endoscopic surgery is one of the main advantages of the endoscopic procedure. The authors stipulate that intradiscal placement of the working cannula during the endoscopic decompression has the potential for less surgical trauma to the already irritated neural elements and may impact postoperative outcomes. The authors are currently investigating whether that is or is not the case. Another clinically relevant question worth investigating in future studies is whether or not the endoscopic decompression can remove more significant amounts of herniated disc tissue from the symptomatic LDH level causing the CES because of the direct access and better visualization of the offending compressive pathology than in an open laminectomy. At a minimum, the authors of this study demonstrated that the mere presence of CES is not a contraindication to employing the full endoscopic decompression technique. While different standards of care exist in Asia, Europe, and the Americas with respect to accepted best practices of surgical CES care, it is clear from this article that clinical results with full endoscopic CES decompression depend on patient selection and surgeons' skill level. Its best indications need to be further studied.

\section{REFERENCES}

1. Fraser S, Roberts L, Murphy E. Cauda equina syndrome: a literature review of its definition and clinical presentation. Arch Phys Med Rehabil. 2009;90(11):1964-1968.

2. Ma B, Wu H, Jia LS, Yuan W, Shi GD, Shi JG. Cauda equina syndrome: a review of clinical progress. Chin Med $\mathrm{J}$ (Engl). 2009;122(10):1214-1222.

3. Busse JW, Bhandari M, Schnittker JB, Reddy K, Dunlop RB. Delayed presentation of cauda equina syndrome secondary to lumbar disc herniation: functional outcomes and healthrelated quality of life. CJEM. 2001;3(4):285-291.

4. Shi J, Jia L, Yuan W, et al. Clinical classification of cauda equina syndrome for proper treatment. Acta Orthop. 2010;81(3):391-395.

5. Srikandarajah N, Boissaud-Cooke MA, Clark S, Wilby 
MJ. Does early surgical decompression in cauda equina syndrome improve bladder outcome? Spine (Phila Pa 1976). 2015;40(8):580-583.

6. Todd NV. Letter to the editor concerning "Cauda Equina Syndrome treated by surgical decompression: the influence of timing on surgical outcome" by A. Qureshi, P. Sell (2007) Eur Spine J. 16:2143-2151. Eur Spine J. 2009;18(9):1391-1392; author reply 1393 .

7. Aly TA, Aboramadan MO. Efficacy of delayed decompression of lumbar disk herniation causing cauda equina syndrome. Orthopedics. 2014;37(2):e153-156.

8. Gleave JR, MacFarlane R. Prognosis for recovery of bladder function following lumbar central disc prolapse. $\mathrm{Br} J$ Neurosurg. 1990;4(3):205-209.

9. Dinning TA, Schaeffer HR. Discogenic compression of the cauda equina: a surgical emergency. Aust $N$ Z J Surg. 1993;63(12):927-934.

10. Olivero WC, Wang H, Hanigan WC, et al. Cauda equina syndrome (CES) from lumbar disc herniations. J Spinal Disord Tech. 2009;22(3):202-206.

11. Shapiro S. Cauda equina syndrome secondary to lumbar disc herniation. Neurosurgery. 1993;32(5):743-746; discussion 746-747.

12. Kim HS, Kim SW, Lee SM, Shin H. Endoscopic discectomy for the cauda equina syndrome during third trimester of pregnancy. J Korean Neurosurg Soc. 2007;42(5):419-420.

13. Jha SC, Tonogai I, Takata Y, et al. Percutaneous endoscopic lumbar discectomy for a huge herniated disc causing acute cauda equina syndrome: a case report. J Med Invest. 2015;62(1-2):100-102.

14. Qureshi A, Sell P. Cauda equina syndrome treated by surgical decompression: the influence of timing on surgical outcome. Eur Spine J. 2007;16(12):2143-2151.

15. Yeung AT, Tsou PM. Posterolateral endoscopic excision for lumbar disc herniation: Surgical technique, outcome, and complications in 307 consecutive cases. Spine (Phila Pa 1976). 2002;27(7):722-731.
16. Li X, Dou Q, Hu S, et al. Treatment of cauda equina syndrome caused by lumbar disc herniation with percutaneous endoscopic lumbar discectomy. Acta Neurol Belg. 2016;116(2):185-190.

17. Jensen RL. Cauda equina syndrome as a postoperative complication of lumbar spine surgery. Neurosurg Focus. 2004;16(6):e7.

18. Hussain SA, Gullan RW, Chitnavis BP. Cauda equina syndrome: outcome and implications for management. $\mathrm{Br} J$ Neurosurg. 2003;17(2):164-167.

19. McCarthy MJ, Aylott CE, Grevitt MP, Hegarty J. Cauda equina syndrome: factors affecting long-term functional and sphincteric outcome. Spine (Phila Pa 1976). 2007;32(2):207-216.

Disclosures and COI: The views expressed in this article represent those of the authors and no other entity or organization. The authors have no conflict of interest in regard to this research.

Corresponding Author: Kai-Uwe Lewandrowski, MD, Center for Advanced Spine Care of Southern Arizona and Surgical Institute of Tucson, 4787 E Camp Lowell Road, Tucson, AZ 85712. Phone: (520) 204-1445; Fax: (520) 407-6740; Email: business@tucsonspine.com.

Published 26 February 2021

This manuscript is generously published free of charge by ISASS, the International Society for the Advancement of Spine Surgery. Copyright (C) 2021 ISASS. To see more or order reprints or permissions, see http://ijssurgery.com. 